\title{
Counting BPS states of the M-5brane
}

\author{
Giulio Bonelli ${ }^{1}$ \\ ${ }^{1}$ Physique Theorique et Mathematique - Universite Libre de Bruxelles \\ Campus Plaine C.P. 231 - B 1050 Bruxelles, Belgium \\ and \\ Theoretische Natuurkunde - Vrije Universiteit Brussel \\ Pleinlaan, 2 - B 1050 Brussel, Belgium \\ bonelli@phys.uu.nl
}

\begin{abstract}
An on-shell model for the supersymmetric index counting multiplicities of BPS states of the M5-brane theory is reviewed. In particular we explicitly study the tensionless Little String states appearing at intersections in a bound state of $N$ 5-branes wrapped on a six-manifold with product topology $M_{4} \times T^{2}$.
\end{abstract}

\section{Introduction}

A proper definition of M-theory as a non perturbative framework for superstring theories is still an open problem. It is strongly shaded, among other problems, by a widely incomplete knowledge of the very structure of the world-volume theory of the M5-branes.

A conjectural formulation of the M5-brane theory has been given in [1] in terms of a six-dimensional string theory, called Little String Theory (LST), which should describe the world-volume theory of the M5-brane. This arises naturally once we understand the M-theory 5-brane not only as the magnetic dual of the membrane but also as Dirichlet surface for membranes. This picture is natural from the point of view of the equivalence of M-theory compactification on a circle $S^{1}$ and type IIA string theory. In fact, under $S^{1}$ compactification, the M5-brane generates both the NS-5branes which are magnetic duals to fundamental strings and the D4-branes on which fundamental strings can end. At the same time the fundamental strings are generated by membranes wrapping the circle.

Despite the simplicity and naturalness of the above picture, LST shows several peculiar features which make its actual implementation via the usual tools of superstring theory, to say the least, tricky.

A proper analysis [0] of the boundary degrees of freedom of the membranes ending on the 5-brane gives as a result that the low energy spectrum of LST is given by the $(0,2)$ self-dual tensor multiplet. Its bosonic content is given by a 2 -form with self-dual curvature which couples minimally to the strings and 5 bosons in the vector representation of the local $S O(5)$ group describing the transverse dispalcements of the 5-brane in the 11 dimensional target space of M-theory (i.e. these 5 bosons are a section of the normal bundle of the 5-brane world-volume embedded in 11 dimensions). 
A first consequence of this analysis is that LST should not be understood in the usual perturbative terms. It is, because of self-duality, a symmetric dyon with equal electric and magnetic charge and, applying the Dirac quantization rule $e g^{\prime}+g e^{\prime}=2 \pi n$, we find $e^{2}=\pi n$. This means that the elementary charge $e_{0}=\sqrt{\pi}$ is not an adjustable parameter and therefore, assuming that the charge is a non constant function of the string coupling, it is natural to expect that LST does not admit a usual perturbative world-sheet formulation. Another unusual feature is the LST target space dimension which is 6 and this means that LST does not fulfill the criticality bound as a string theory. Moreover a further possibly problematic aspect is given by its unusual non-gravitational low energy spectrum.

Notwithstanding the open questions concerning a proper off-shell formulation of LST, it is possible to study some of its on-shell properties by indirect methods and to check the validity of the above model.

The specific issue we are going to treat here is a counting of BPS multiplets of states in LST. These are encoded in the generalized supersymmetric Witten index of the theory. We will propose an on-shell framework to calculate these objects within a six dimensional perspective on a world-volume six manifold of the product form $T^{2} \times M_{4}$. The particular form of the six-manifold allows the use of a duality map between M-theory on $T^{2}$ and type IIB theory in the limit of vanishing $T^{2}$-volume to check the validity of our results. Under this duality map, M5-branes wrapped on the above product manifold correspond to D3-branes wrapped on $M_{4}$. This map therefore reverts our problem to a calculation of a supersymmetric path integral in a corresponding suitable four-dimensional gauge theory whose coupling is identifyed with the $T^{2}$ modular parameter.

The result of our calculation will be to explicitly recognize the presence of tensionless string states in correspondence with the intersection between different 5-branes in a given bound state and to give a precise formula to count their multiplicities.

This talk is mainly based on [3, 4].

\section{The M5-branes index on $T^{2} \times M_{4}$}

To properly embed our problem in M-theory, we consider M-theory on $W=Y_{6} \times T^{2} \times R^{3}$, where $Y_{6}$ is a Calabi-Yau threefold of general holonomy. Let $M_{4}$ be a supersymmetric simply connected four-cycle in $Y_{6}$ which we take to be smooth. Under these assumptions $M_{4}$ is automatically equipped with a Kaehler form $\omega$ induced from $Y_{6}$ and is simply connected. We consider then $N$ M5-branes wrapped around $C=T^{2} \times M_{4}$.

It can be shown that in this specific geometrical set-up [4] the potential anomalies which tend to ruin gauge invariance of the world-volume theory are absent and that it is then meaningful to define a supersymmetric index for the above 5-branes bound states by extending the approach in [5]. As it is well known, the supersymmetric index is independent on smooth continue parameters and as a consequence we have that this counting of supersymmetry preserving states have to be independent on the $T^{2}$ volume.

In [4] the calculation of the supersymmetric index was performed in the limiting cases of small and of large volumes of the $T^{2}$ and shown to agree while in [3] a six dimensional framework was proposed which reproduces the above calculations as a one loop string supersymmetric path-integral. We will review the last construction.

Let us start with the single M5-brane case. The bosonic spectrum of the low energy world-volume theory of this 5-brane is given by a 2 -form $V$ with self-dual curvature and five real bosons taking values in the normal bundle $N_{C}$ induced by the structure of the embedding as $\left.T_{W}\right|_{C}=T_{C} \oplus N_{C}$. Passing to the holomorphic part and to the 
determinants and using the Calabi-Yau nature of $Y_{6}$, it follows that the five transverse bosons are respectively, three non-compact real scalars $\phi_{i}$ and one complex section $\Phi$ of $K_{M_{4}}=\Lambda^{-2} T_{M_{4}}^{(1,0)}$, which is the canonical line bundle of $M_{4}$. A (partially) twisted chiral $(0,2)$ supersymmetry of the type considered in [6] completes the spectrum. It is given by a doublet of complex anti-commuting fields which are $(2,0)$-forms in six dimensions and a doublet of complex anti-commuting fields which are scalars in six dimensions.

The calculation of the supersymmetric index for this part of the spectrum can be done with path-integral techniques. It is classically exact because of boson/fermion exact cancellation and it results in a zero-modes amplitude for the self-dual tensor field. This can be analyzed following the results of [7]. It consists of a $\theta$-function of the lattice of the self-dual harmonic three forms. The $\theta$-function is not completely specified in [7] because of the possible inequivalent choices of its characteristics $\left[\begin{array}{c}\alpha \\ \beta\end{array}\right]$. It is

$$
\theta\left[\begin{array}{l}
\alpha \\
\beta
\end{array}\right]\left(Z^{0} \mid 0\right)=\sum_{k} e^{i \pi\left((k+\alpha) Z^{0}(k+\alpha)+2(k+\alpha) \beta\right)},
$$

where $Z^{0}$ is a period matrix of the relevant six-manifold cohomology that we specify shortly. Let $\left\{E^{(6)}, \tilde{E}^{(6)}\right\}$ be a symplectic basis of harmonic 3 -forms on the six-manifold at hand such that, in matrix notation,

$$
\int E^{(6)} E^{(6)}=0, \quad \int \tilde{E}^{(6)} E^{(6)}=1, \quad \int \tilde{E}^{(6)} \tilde{E}^{(6)}=0 .
$$

We can expand $\tilde{E}^{(6)}=X^{0} E^{(6)}+Y^{0 *} E^{(6)}$, where ${ }^{*}$ is the Hodge operator. Then $Z^{0}$ is defined as $Z^{0}=X^{0}+i Y^{0}$. In general, under modular transformations (i.e. global diffeomeorphisms of the 5 -brane world-volume) these $\theta$-functions transform among each others. If we ask for a single candidate closed under modular transformations, we see [8] that the natural choice is given by $\left[\begin{array}{l}\alpha \\ \beta\end{array}\right]=\left[\begin{array}{l}0 \\ 0\end{array}\right]$.

In our case the world volume is in the product form $T^{2} \times M_{4}$ and [3] we have $\frac{1}{2} b_{3}\left(T^{2} \times M_{4}\right)=$ $b_{2}\left(M_{4}\right)$ and $Z^{0}=-\tau^{(1)} Q+i \tau^{(2)} 1$, where $Q$ is the intersection matrix on $M_{4}, 1$ is the unity matrix and $\tau=\tau^{(1)}+i \tau^{(2)}$ is the modulus of the $T^{2}$. The relevant $\theta$-function is then

$$
\Theta\left(Z^{0}\right) \equiv \theta\left[\begin{array}{l}
0 \\
0
\end{array}\right]\left(Z^{0} \mid 0\right)=\sum_{k} e^{i \pi k Z^{0} k}=\sum_{m \in \Lambda} q^{\frac{1}{4}(m, * m-m)} \bar{q}^{\frac{1}{4}(m, * m+m)} \equiv \theta_{\Lambda}(q, \bar{q})
$$

where $q=e^{2 i \pi \tau}$ and $\Lambda$ is the lattice of integer period elements in $H^{2}\left(M_{4}, \mathbf{R}\right)$.

To count the full BPS spectrum of the theory a second sector is still lacking. In fact, the 5-brane theory is completed in the UV by the little string theory which has BPS saturates strings which eventually have to be kept into account in the calculation of the complete supersymmetric index. Even if a full off-shell model for this six-dimensional string theory is not available at the moment, it is possible to follow an on-shell simple calculation scheme for their contribution to the supersymmetric index.

As the susy index is given by a trace on the string Hilbert space, it corresponds to a one-loop string path integral. Moreover, as it is usual in these index calculations, the semiclassical approximation is exact. Now, since $M_{4}$ is simply connected, the only contributions to this path integral can arise from string world sheets wrapping the $T^{2}$ target itself. Therefore the configuration space of $n$ of these string world-sheets $\square$ will be given by the symmetric product $\left(M_{4}\right)^{n} / S_{n}$ whose points parametrize the transverse positions.

\footnotetext{
${ }^{1}$ We are assuming here that only one type of BPS strings has to be counted. This is supported also by the analysis of the short representation of $(0,2)$ supersymmetry in six dimensions given in $[9]$.
} 
Now, since the supersymmetric index calculated the Euler characteristics of the configuration space, we claim that the full contribution from these string BPS configurations is given by

$$
q^{-\chi_{M_{4}} / 24} \sum_{n} q^{n} \chi\left(M_{4}^{n} / S_{n}\right)=q^{-\chi_{M_{4}} / 24} \prod_{n>0} \frac{\left(1-q^{n}\right)^{b_{\text {odd }}}}{\left(1-q^{n}\right)^{b_{\text {even }}}}=\eta(q)^{-\chi_{M_{4}}}
$$

where we fixed a global multiplicative factor $q^{-\chi_{M_{4}} / 24}$ because of modularity requirements and we used well known results from [10]. In [3] it is shown that this result can be obtained also via a natural generalization of the construction done in 11] for toroidal and K3 compactifications to the generic simply connected Kahler case.

The complete supersymmetric index for a single five-brane on $T^{2} \times M_{4}$ is then given multiplying the factor (1) and the factor (2) and reads

$$
\mathcal{I}_{1}^{T^{2} \times M_{4}}=\frac{\theta_{\Lambda}}{\eta^{\chi}} .
$$

We now pass to the analysis of the multi 5-brane case. We will start assuming that the BPS multi five-brane bound states are classified by holomorphic branched coverings of the relevant world volume manifold and by a choice of spin structure on it. Notice that this will result in recostructing exactly the relevant supersymmetric index as it can be calculated from the zero $T^{2}$-volume limit. In this limit [3, 4] we find in fact the four dimensional gauge theory corresponding to a dual multi D3-brane bound state in type IIB and the calculation is under full control In [3] also an independent duality argument is given which supports this picture when the six manifold admits a regular $S^{1}$ fibration. Suppose therefore that we are dealing with a BPS bound state of $N$ 5-branes wrapped on a six manifold $\mathcal{C}$. Then we have to consider the space of rank $\mathrm{N}$ holomorphic coverings of $\mathcal{C}$ in the M-theory target space. Generically this space of coverings will be disconnected and reducible into components consisting of connected irreducible coverings of rank less or equal to $N$. Each of these irreducible holomorphic coverings corresponds to an irreducible bound state. Then the general structure formula for the supersymmetric index of an irreducible bound state of $N$ 5-branes is given by the lifting to the spectral cover of the index formula. In formulas, if $\mathcal{C}_{N}$ is a generic holomorphic covering of $\mathcal{C}$, then $\mathcal{C}_{N}=\cup_{j} \mathcal{C}_{j}^{\text {irr }}$, where $\sum j=N$ and $\mathcal{C}_{j}^{\text {irr }}$ is irreducible. The relative contribution to the index is $\mathcal{I}_{N}^{\mathcal{C}}=\prod_{j} \mathcal{I}_{1}^{\mathcal{C}_{j}^{i r r}}$. This formula has to be understood still schematically since we didn't specified the sum over the spin structures. In the case $\mathcal{C}=T^{2} \times M_{4}$, a generic irreducible holomorphic covering is of the form $\tilde{T}^{2} \times \Sigma_{r}$, where $\tilde{T}^{2}$ is a torus with modulus $\tilde{\tau}=(a \tau+b) / d$ which covers $a \cdot d=n_{r}$ times the original $T^{2}$ and $d>b \geq 0$ and $\Sigma_{r}$ is a rank $r$ holomorphic branched covering of $M_{4}$. The above multiplicities are of course constrained by $n_{r} \cdot r=j$ which is the rank of the six dimensional irreducible covering $\mathcal{C}_{j}^{\text {irr }}$ above. The full supersymmetric index is then given by summing all along the whole set of possible covering structures. Let us perform the sum with respect to the $T^{2}$ coverings first. To do it by preserving modularity, we multiply each factor by a rescaling term $d^{-w-\bar{w}}$, where $(w, \bar{w})$ are the modular weights of $\left.\mathcal{I}\right|_{n_{r}=1}$, and then we sum over all the triples $(a, b, d)$ fulfilling the above condition. This operation coincides exactly with the definition of the Hecke modular operator $\mathcal{H}_{n_{r}}$ and our partial sum now reads $\mathcal{I}_{\left(n_{r}, r\right)}^{T^{2} \times M_{4}}=\mathcal{H}_{n_{r}} \mathcal{I}_{(1, r)}^{T^{2} \times M_{4}}$. By including the sum over the appropriate spin structures and explicitating the dependence over the given irreducible rank $r$ covering $\Sigma_{r}$, we finally get

$$
\mathcal{I}_{n_{r}, r}=\mathcal{H}_{n_{r}} \sum_{\varepsilon} \frac{\theta_{\Lambda^{\Sigma_{r}}+x}}{\eta^{\chi_{\Sigma_{r}}}}
$$


where $\varepsilon$ is a label for the square-roots of the canonical line bundle (spin structures) on $M_{4}$ with respect to a given one as $\mathcal{O}_{\varepsilon} \otimes K^{1 / 2}$ with $\mathcal{O}_{\varepsilon}^{2}=1, x=\left[\mathcal{O}_{\varepsilon}^{\otimes a+1}\right]$ shifts correspondingly the lattice of integer periods $\Lambda^{\Sigma_{a}}$ on $H^{2}\left(\Sigma_{a}, R\right)$.

As it was already mentioned in the introduction, the multi 5-brane interacting model we have in mind consists in a picture where the 5-branes, beside being magnetic duals of the membranes, are also Dirichlet hypersurfaces for them, the boundaries of the membranes being strings in the 5-brane world-volume. In this picture one expects that tensionless BPS string states appear when two or more 5-branes intersect each other. In our geometric picture this is the branching locus of the covering six manifold. Indeed the supersymmetric index (3) reveals clearly the apparing of these extra BPS string states. In our particular case, the $T^{2}$ holomorphic self-covering is unbranched and the little strings contribution is explicitly exposed in the Dedekind $\eta$-function. In fact it enters in the form $\left(\frac{1}{\eta}\right)^{\chi \Sigma_{r}}$, where $\Sigma_{r}$ is a rank $r$ holomorphic covering of $M_{4}$. The explicit dependence on the branching locus or the covering $B_{r}$ appears once we apply the Hurwitz formula $\chi_{\Sigma_{r}}=r \cdot \chi_{M_{4}}-\chi_{B_{r}}$ which holds for the covering structure. We find therefore that

$$
\eta^{-\chi_{\Sigma_{r}}}=\left[\eta^{-\chi_{M_{4}}}\right]^{r} \cdot \eta^{\chi_{B_{r}}}
$$

We read this formula for multiplicities of massless BPS string states as a superposition of the independent BPS states pertaining to each brane copy which is corrected by a further term corresponding to the interacting theory.

\section{Conclusions and Open Problems}

In this talk we have reviewed a six dimensional framework for the evaluation of the supersymmetric index of M-theory five-branes on $T^{2} \times M_{4}$ based on a on shell model for BPS states in LST. The appearing of extra massless BPS states in coincidence with 5 -branes intersection is clearly encoded as a by-product in our formulas.

As we explained in the previous section, the geometric model encoding the structure of the BPS bound states of 5-branes we refer to is based on the idea that the relevant informations about them are encoded in the (geometric) moduli space of branched holomrphic coverings of the six manifold on which the system lives. Although we have different arguments to justify this model, a proof internal to the six dimensional interacting theory is still lacking (together with the off-shell model itself) and it sounds - see how these structures appear in D-brane physics [12, 13] - that the direct solution of this specific point will be available only once the 5-brane (non-local) analogous geometrical structure to the D-branes gauge bundle structure will be given.

Moreover, the above formula for the supersymmetric index (lets consider for simplicity a single 5-brane) should generalize to

$$
\Theta\left(Z_{\mathcal{C}}^{0}\right) / \mathrm{N}\left(Z_{\mathcal{C}}^{0}\right)
$$

for a 5 -brane on a generic supersymmetric six cycle $\mathcal{C}$ where $\mathrm{N}\left(Z_{\mathcal{C}}^{0}\right)^{-1}$ has to be a modular function of the period matrix $Z_{\mathcal{C}}^{0}$ of the six manifold which reduces to $\eta(\tau)^{-\chi_{M_{4}}}$ in the case $\mathcal{C}=T^{2} \times M_{4}$ and which calculates the BPS multiplicities of LS states in the general case.

Another interesting point would be to generalize the above treatment to amplitudes of BPS (surface) operators of the type considered in [14]. 
The interested reader is referred to [15] for further developments in the subjects treated here.

Acknowledgments I would like to warmly thank M. Bertolini for interesting discussions, the Departments of Physics of Padua and Turin Universities where these results have been presented and the organizers of the Corfu' RTN meeting. Work supported by the European Commission RTN programme HPRN-CT-2000-00131 as subcontractor of Leuven University.

\section{References}

[1] A. Strominger, "Open P-Branes", Phys. Lett. B383 (1996) 44-47 [hepth/9512059].

[2] K. Becker and M. Becker, "Boundaries in M-Theory", Nucl.Phys. B472 (1996) 221230 hep-th/9602071.

[3] G. Bonelli, "On the Supersymmetric Index of the M-theory 5-brane and Little String Theory", Phys. Lett. B521/3-4 (2001) 383-390 [hep-th/0107051].

[4] G. Bonelli, "The geometry of the M5-brane and TQFTs", J.Geom.Phys. 40 (2001) 13-25 [hep-th/0012075].

[5] J. A. Minahan, D. Nemeschansky, C. Vafa and N. P. Warner, "E-strings and N = 4 topological Yang-Mills theories" Nucl. Phys. B527 (1998) 581 hep-th/9802168.

[6] L. Baulieu and P. West, "Six-dimensional TQFTs and twisted supersymmetry" Phys. Lett. B436 (1998) 97 hep-th/9805200.

[7] M. Henningson, B. E. Nilsson and P. Salomonson, "Holomorphic factorization of correlation functions in $(4 \mathrm{k}+2)$-dimensional (2k)-form gauge theory," JHEP 9909 (1999) 008 hep-th/9908107.

[8] A. Gustavsson, "On the holomorphically factorized partition function for abelian gauge theory in six dimensions", hep-th/0008161.

[9] A. Gustavsson and M. Henningson, "A short representation of the six-dimensional $(2,0)$ algebra" JHEP 0106 (2001) 054 hep-th/0104172.

[10] C. Vafa and E. Witten, "A Strong coupling test of S duality", Nucl. Phys. B431 (1994) 3 hep-th/9408074.

[11] R. Dijkgraaf, E. Verlinde and H. Verlinde, "BPS quantization of the five-brane", Nucl. Phys. B 486 (1997) 89 hep-th/9604055.

[12] M. Bershadsky, V. Sadov, C. Vafa, "D-Branes and Topological Field Theories" Nucl.Phys. B463 (1996) 420-434 hep-th/9511222;

[13] G. Bonelli, L. Bonora, S. Terna and A. Tomasiello, "Instantons and scattering in $\mathrm{N}=4 \mathrm{SYM}$ in 4D" hep-th/9912227.

[14] M. Henningson, "Commutation relations for surface operators in six-dimensional $(2,0)$ theory," JHEP 0103 (2001) 011 [hep-th/0012070].

[15] G. Bonelli, "The M5-brane on K3 and del Pezzo's and multi-loop string amplitudes" hep-th/0111126. 